\title{
Torsion of Truncated Hollow Spherical Elastic Body
}

\author{
I. ECSEDI ${ }^{1}$, Á. J. LENGYEL ${ }^{2}$, A. BAKSA ${ }^{3}$, D. GÖNCZI ${ }^{4}$ \\ 1,2,3,4 University of Miskolc, Institute of Applied Mechanics \\ ${ }^{1}$ mechecs@uni-miskolc.hu, ${ }^{2}$ mechlen@uni-miskolc.hu, ${ }^{3}$ mechab@uni-miskolc.hu, ${ }^{4}$ mechgoda@uni-miskolc.hu
}

Abstract. This paper deals with the torsion of a body of rotation whose shape is a truncated hollow sphere. The material of the truncated hollow sphere is isotropic, homogeneous and linearly elastic. To solve the torsion problem, the theory of torsion of shafts of varying circular cross section is used, which is introduced by Michell and Föppl. Analytical solution is given for the shearing stresses and displacements. A numerical example illustrates the application of the presented solution. The results of the presented numerical example can be used as a benchmark problem to verify the accuracy of the results computed by finite element simulations.

Keywords: Torsion, body of rotation, truncated hollow sphere, elasticity

\section{Introduction}

The torsional deformation of a body of rotation is a very important chapter in the mechanics of structures $[1,2,3,4,5,6]$. Paper by Bramble deals with a thick elastic spherical shell subjected to concentrated torques at the end-points of a diameter of the outer spherical boundary surface [7]. It is proven that the solution is uniquely determined and an explicit expression is derived for it. The author of [7] uses the fact that the stress function is closely related to an axially symmetric harmonic function defined in a seven-dimensional space. Pöschl studied the torsion problem of circular conical elastic body [8]. Book written by Arutyunyan and Abramyan [9] gives an analytical solution for the torsion of a hemisphere. For body of rotations, whose boundary surfaces are coordinate surfaces of an orthogonal curvilinear coordinate system, the closed form solutions are derived to the torsional boundary value problem [11]. There are several works on the problem of torsion of a body of rotation. It is not the purpose of this paper to provide a detailed list of the literature on this topic. The book of Arutyunyan and Abramyan [9], and the book of Lekhnitskii [12] present many works on the torsion of elastic shafts of varying circular cross section. In this paper the torsion of the 'hollow' truncated sphere is studied. The considered truncated sphere is made of homogeneous, isotropic and linearly elastic material. The formulation of the torsional boundary value problem is given in cylindrical coordinate system $\operatorname{Or} \varphi z$ (Fig. 1). The meridian section of the truncated hollow sphere is shown in Fig. 2. The truncated hollow sphere occupies the space domain $B$ in the three-dimensional space

$$
B=\left\{(r, \varphi, z) \mid 0 \leq z \leq L, r_{1}(z)=\sqrt{R_{1}^{2}-z^{2}} \leq \mathrm{r} \leq r_{2}(z)=\sqrt{R_{2}^{2}-z^{2}}, 0 \leq \varphi \leq 2 \pi\right\} .
$$




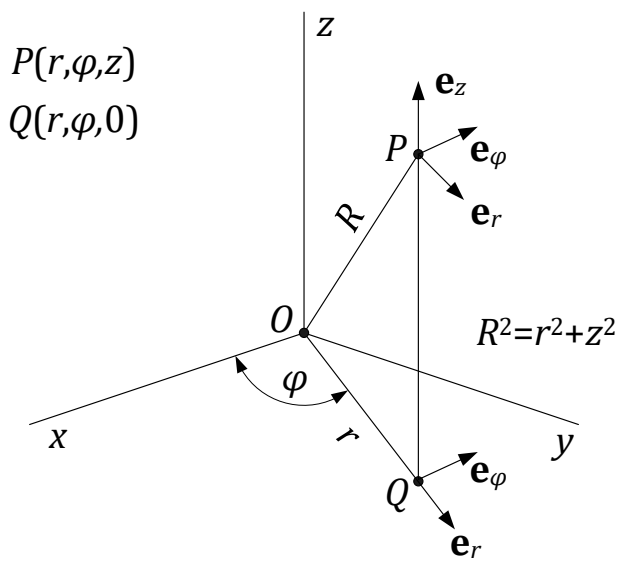

Figure 1. Cylindrical coordinates $(r, \varphi, z)$

The boundary curve of the meridian section $A$ of body $B$

$$
\partial A=\partial A_{1} \cup \partial A_{2} \cup \partial A_{3} \cup \partial A_{4}
$$

as shown in Fig. 2. The boundary surface segments $\partial B_{1}$ and $\partial B_{2}$ are stress free. The tangential traction $\tau_{\varphi z}$ is not point-wisely prescribed on the end cross sections $\partial B_{3}$ and $\partial B_{4}$, only the moments of tractions at the end cross sections about axis $z$ are given. Denote $T$ the applied torque on end cross sections $\partial B_{3}$ and $\partial B_{4} . \partial B_{i}$ is obtained from a complete rotation of $\partial A_{i}(i=1,2,3,4)$ about axis $z$. According to the condition of mechanical equilibrium we have

$$
T=\int_{\partial B_{3}} r \tau_{\varphi Z}(r, \varphi, 0) \mathrm{d} a=\int_{\partial B_{4}} r \tau_{\varphi Z}(r, \varphi, L) \mathrm{d} a,
$$

where $\mathrm{d} a$ denotes the area element on the boundary surface $\partial B$. Equation (3) formulates a weak traction boundary condition. This traction boundary value problem when the curved boundary part of the truncated hollow sphere is traction free and satisfies the weak boundary condition (3) can have solutions that differ a rigid body motion.

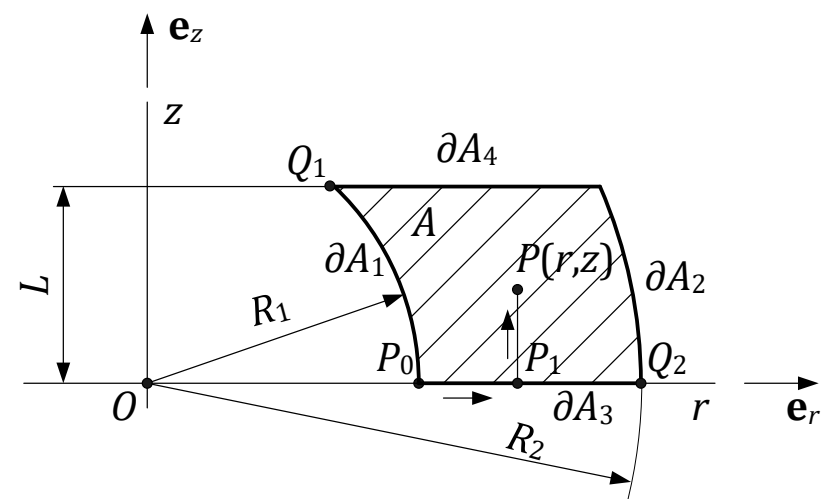

Figure 2. Meridian section of the truncated hollow sphere

\section{Formulation of the problem}

The Michell-Föppl's theory of the torsion of shafts of varying circular cross section is based on the next displacement field $[2,3,4,10]$ 


$$
\mathbf{u}(r, \varphi, z)=v(r, z) \mathbf{e}_{\varphi}(\varphi), \quad(r, \varphi, z) \in B
$$

The non-zero infinitesimal strains are

$$
\gamma_{r \varphi}=\frac{\partial v}{\partial r}-\frac{v}{r}, \quad \gamma_{z \varphi}=\frac{\partial v}{\partial z}
$$

where $\gamma_{r \varphi}$ and $\gamma_{z \varphi}$ are shearing strains on the plane $\varphi=$ constant. For isotropic homogeneous Hookean material the shearing stresses are given by

$$
\tau_{r \varphi}=G \gamma_{r \varphi}=G r \frac{\partial \psi}{\partial r}, \quad \tau_{z \varphi}=G \gamma_{z \varphi}=G r \frac{\partial \psi}{\partial z}, \quad \psi=\frac{v(r)}{r} .
$$

In the above equation $G$ denotes the shear modulus of a homogeneous, isotropic and linearly elastic material. Since there are not body forces, the only non-trivial equation of equilibrium is

$$
\frac{\partial \tau_{r \varphi}}{\partial r}+\frac{\partial \tau_{z \varphi}}{\partial z}+\frac{2 \tau_{r \varphi}}{r}=0 .
$$

It is known that the general solution of equilibrium equation (7) in terms of first order stress function $U=U(r, z)$ can be represented as

$$
r^{2} \tau_{r \varphi}=-\frac{\partial \mathrm{U}}{\partial z}, \quad r^{2} \tau_{z \varphi}=\frac{\partial \mathrm{U}}{\partial r} .
$$

Combination of equation (6) with equation (8) gives the strain compatibility equation in terms of stress function $U=U(r, z)$

$$
\frac{\partial^{2} U}{\partial r^{2}}-\frac{3}{r} \frac{\partial U}{\partial r}+\frac{\partial^{2} U}{\partial z^{2}}=0, \quad(r, z) \in A .
$$

The curved boundary surfaces $\partial B_{i}(i=1,2)$ are traction free, that is

$$
\tau_{r \varphi}(r, z) n_{r}+\tau_{z \varphi}(r, z) n_{z}=-\frac{n_{r}}{r^{2}} \frac{\partial U}{\partial z}+\frac{n_{z}}{r^{2}} \frac{\partial U}{\partial r}=-\frac{t_{r}}{r^{2}} \frac{\partial U}{\partial z}-\frac{t_{z}}{r^{2}} \frac{\partial U}{\partial r}=-\frac{\partial U}{\partial s}=0 .
$$

In equation (10) $n_{r}, n_{z}$ are the components of unit normal vector $\mathbf{n}$ and the $t_{r}, t_{z}$ are the componens of unit tangential vector $\mathbf{t}$ of the boundary curve $\partial A_{3} \cup \partial A_{4}$ and $s$ is arc-length defined on the boundary curve $\partial A_{3} \cup \partial A_{4}$ (Fig. 3). Equation (10) shows that the stress function is constant on the curved boundary surface segments of the body of rotation. The torque $T$ is taken across by the cross section at $z$ is obtained as

$$
T(z)=2 \pi \int_{r_{1}(z)}^{r_{2}(z)} r^{2} \tau_{z \varphi} \mathrm{d} r=2 \pi\left(U\left(r_{2}(z), z\right)-U\left(r_{1}(z), z\right)\right)=2 \pi U\left(r_{2}(z), z\right) .
$$

In equation (11) we prescribe that

$$
U\left(r_{1}(z), z\right)=\text { constant }=0, \quad 0 \leq z \leq L .
$$

We can do it, since the $U(r, z)$ and $\bar{U}(r, z)=U(r, z)+K$ give the same stress and strain fields. The global condition of the mechanical equilibrium can be formulated as $T(z)=$ constant. 


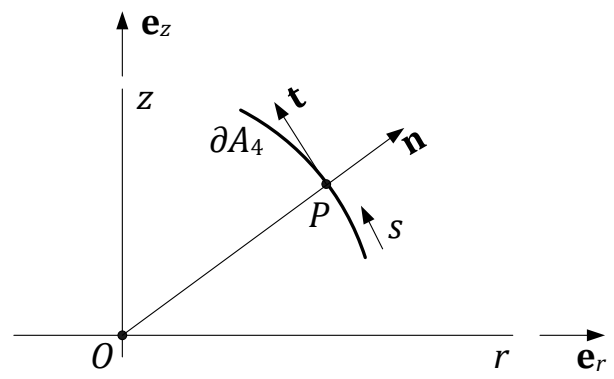

Figure 3. Illustration of tangential and normal unit vectors to the stress free boundary curve

\section{Solution of the torsional problem}

Our aim is to get the solution of the partial differential equation (9) under the boundary conditions

$$
U\left(r_{1}(z), z\right)=0, \quad U\left(r_{2}(z), z\right)=\frac{T}{2 \pi}, \quad 0<z<L .
$$

We introduce a new variable $t$

$$
t=r^{2}+z^{2}, \quad(r, z) \in A \cup \partial A .
$$

Simple computation shows that

$$
\begin{gathered}
\frac{\mathrm{d}^{2} U}{\mathrm{~d} t^{2}} \frac{\partial U}{\partial r}=2 r \frac{\mathrm{d} U}{\mathrm{~d} t}, \quad \frac{\partial^{2} U}{\partial r^{2}}=4 r^{2} \frac{\mathrm{d}^{2} U}{\mathrm{~d} t^{2}}+2 \frac{\mathrm{d} U}{\mathrm{~d} t}, \\
\frac{\partial U}{\partial z}=2 z \frac{\mathrm{d} U}{\mathrm{~d} t}, \quad \frac{\partial^{2} U}{\partial z^{2}}=4 z^{2} \frac{\mathrm{d}^{2} U}{\mathrm{~d} t^{2}}+2 \frac{\mathrm{d} U}{\mathrm{~d} t} .
\end{gathered}
$$

Substitution of equations (15), (16) into the partial differential equation (9) gives an ordinary differential equation for $U=U(t)$

$$
\frac{\mathrm{d}^{2} U}{\mathrm{~d} t^{2}}-\frac{1}{2 t} \frac{\mathrm{d} U}{\mathrm{~d} t}=0
$$

The boundary condition (13) $)_{1,2}$ can be reformulated as

$$
U\left(t_{1}\right)=0, \quad t_{1}=R_{1}^{2}, \quad U\left(t_{2}\right)=\frac{T}{2 \pi}, \quad t_{2}=R_{2}^{2} .
$$

The solution of differential equation under the boundary condition (18) $)_{1,2}$ in terms of $r$ and $z$ is

$$
U(r, z)=\frac{T}{2 \pi} \frac{\left(\sqrt{r^{2}+z^{2}}\right)^{3}-R_{1}^{3}}{R_{2}^{3}-R_{1}^{3}}, \quad(r, z) \in A \cup \partial A .
$$

Application of formulae of shearing stresses $\tau_{r \varphi}$ and $\tau_{z \varphi}$ gives

$$
\tau_{r \varphi}(r, z)=-\frac{3 T}{2 \pi} \frac{z}{r^{2}} \frac{\sqrt{r^{2}+z^{2}}}{R_{2}^{3}-R_{1}^{3}}, \quad \tau_{z \varphi}(r, z)=\frac{T}{2 \pi r} \frac{\sqrt{r^{2}+z^{2}}}{R_{2}^{3}-R_{1}^{3}} .
$$

Resulting shearing stress $\tau_{\varphi}$ can be computed as

$$
\tau_{\varphi}(r, z)=\sqrt{\tau_{r \varphi}^{2}+\tau_{z \varphi}^{2}}=\frac{T}{2 \pi r^{2}} \frac{\sqrt{\left(r^{2}+z^{2}\right)\left(r^{2}+9 z^{2}\right)}}{R_{2}^{3}-R_{1}^{3}} .
$$

Determination of the function $\psi=\psi(r, z)$ is based on equation (6). It is obvious 


$$
\frac{\partial \psi}{\partial r}=-\frac{3 T}{2 \pi G} \frac{z}{r^{3}} \frac{\sqrt{r^{2}+z^{2}}}{R_{2}^{3}-R_{1}^{3}}, \quad \frac{\partial \psi}{\partial z}=\frac{3 T}{2 \pi G r^{2}} \frac{\sqrt{r^{2}+z^{2}}}{R_{2}^{3}-R_{1}^{3}} .
$$

Since we have

$$
\frac{\partial^{2} \psi}{\partial z \partial r}=-\frac{\partial}{\partial z} \frac{3 T}{2 \pi G} \frac{z}{r^{3}} \frac{\sqrt{r^{2}+z^{2}}}{R_{2}^{3}-R_{1}^{3}}=\frac{\partial^{2} \psi}{\partial r \partial z}=\frac{\partial}{\partial r} \frac{3 T}{2 \pi G r^{2}} \frac{\sqrt{r^{2}+z^{2}}}{R_{2}^{3}-R_{1}^{3}},
$$

the formula (24) gives the explicit solution of the system of differential equations (Fig. 2)

$$
\psi(r, z)=\int_{P_{0} P_{1}} \frac{\partial \psi}{\partial r} \mathrm{~d} r+\frac{\partial \psi}{\partial z} \mathrm{~d} z+\int_{P_{1} P} \frac{\partial \psi}{\partial r} \mathrm{~d} r+\frac{\partial \psi}{\partial z} \mathrm{~d} z
$$

A detailed computation gives

$$
\psi(r, z)=\frac{3 T}{4 \pi G\left(R_{2}^{3}-R_{1}^{3}\right)}\left(\frac{z}{r} \sqrt{\left(\frac{z}{r}\right)^{2}+1}+\ln \left(\frac{z}{r} \sqrt{\left(\frac{z}{r}\right)^{2}+1}\right)\right) .
$$

The expression of the circumferential displacement $v=v(r, z)$ is as follows

$$
v(r, z)=\frac{3 T r}{4 \pi G\left(R_{2}^{3}-R_{1}^{3}\right)}\left(\frac{z}{r} \sqrt{\left(\frac{z}{r}\right)^{2}+1}+\ln \left(\frac{z}{r} \sqrt{\left(\frac{z}{r}\right)^{2}+1}\right)\right) .
$$

It is evident the cross section at $z=0$ is fixed, that is

$$
\psi(r, 0)=0, \quad v(r, 0)=0 .
$$

From equations (6) and (8) it follows that

$$
\frac{\partial U}{\partial z}=-G r^{3} \frac{\partial \psi}{\partial r}, \quad \frac{\partial U}{\partial r}=G r^{3} \frac{\partial \psi}{\partial z} .
$$

Since

$$
\frac{\partial^{2} U}{\partial r \partial z}=-G r^{3} \frac{\partial^{2} \psi}{\partial r^{2}}=\frac{\partial^{2} U}{\partial z \partial r}=G r^{3} \frac{\partial^{2} \psi}{\partial z^{2}}, \quad(r, z) \in A \cup \partial A
$$

the function $\psi=\psi(r, z)$ satisfies the partial differential equation written below, in the domain $A$

$$
\frac{\partial^{2} \psi}{\partial r^{2}}+\frac{3}{r} \frac{\partial \psi}{\partial r}+\frac{\partial^{2} \psi}{\partial z^{2}}=0, \quad(r, z) \in A
$$

The stress free boundary condition on the curved boundary is expressed as

$$
r \frac{\partial \psi}{\partial r}+z \frac{\partial \psi}{\partial z}=0, \quad(r, z) \in \partial A_{1} \cup \partial A_{2}
$$

Here, it is used

$$
\begin{gathered}
n_{r}=\frac{r}{R_{i}}, \quad n_{z}=\frac{z}{R_{i}}, \quad(r, z) \in \partial A_{i}, \quad(i=1,2), \\
\tau_{r \varphi}=G r^{3} \frac{\partial \psi}{\partial r}, \quad \tau_{z \varphi}=G r^{3} \frac{\partial \psi}{\partial z} .
\end{gathered}
$$

\section{Numerical example}

In the numerical example the following data are used $R_{1}=0.036 \mathrm{~m}, R_{2}=0.075 \mathrm{~m}, L=0.03 \mathrm{~m}, G=$ $1.6 \times 10^{8} \mathrm{~Pa}, T=10 \mathrm{kNm}$. The contour lines of the stress function $U=U(r, z)$ and the function $\psi=$ $\psi(r, z)$ are shown in Fig. 4 and Fig. 5. The resulting shearing stress reaches its maximum value at point 
$Q_{1}$ of the cross section and the minimum value of the resulting shearing stress is at point $Q_{2}$ of the cross section (Fig. 2). From formula (21) we get

$$
\tau_{\varphi}\left(Q_{1}\right)=35.5427 \mathrm{MPa}, \quad \tau_{\varphi}\left(Q_{2}\right)=4.2416 \mathrm{MPa} .
$$

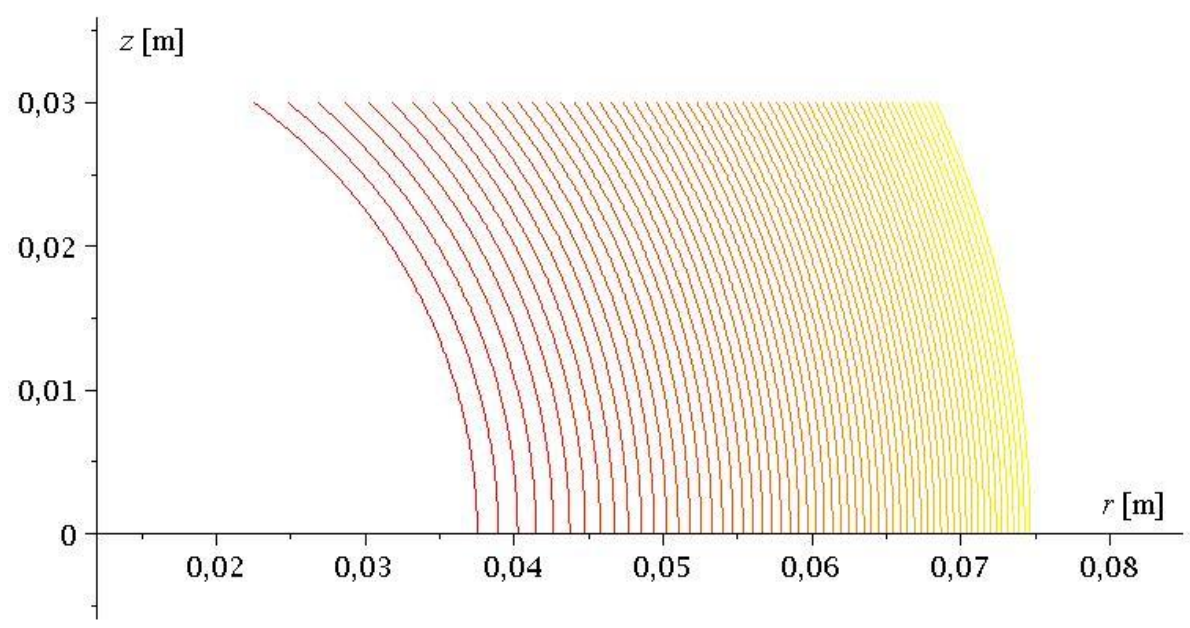

Figure 4. Contour lines of stress function $U=U(r, z)$

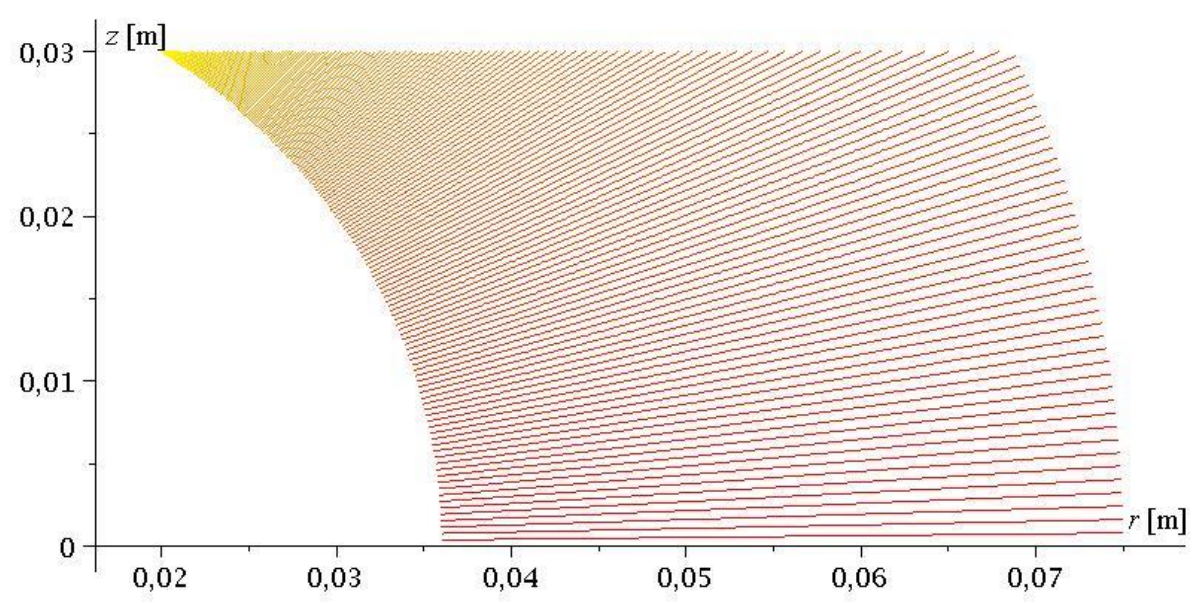

Figure 5. Contour lines of function $\psi=\psi(r, z)$

\section{Conclusions}

An analytical solution is presented for the torsion problem of truncated hollow spherical body whose material is isotropic, homogeneous and linearly elastic. It is assumed that the deformations are small and the formulation of linearized theory of elasticity can be used. Numerical example illustrates the applications of the derived formulae.

\section{References}

[1] Saada, A. S. (1974) 'Elasticity. Theory and its Applications' London: Pergamon Press Inc., doi: 10.1016/C2013-0-02524-8. 
[2] Sokolnikoff, I. S. (1956) 'Mathematical Theory of Elasticity'. New York: McGraw-Hill. doi: $10.2307 / 3608899$.

[3] Timoshenko, S. P., Goodier, J. N. (1970) 'Theory of Elasticity'. (3rd edition) New York: McGrawHill.

[4] Szoljanik Krassza, K. V. (1949) 'Torsion of Shafts of Varying Circular Cross Section', (in Russian), Moscow: Gosztehizdat.

[5] Renton, J. D. (2000) 'Elastic Beams and Frames', Oxford: Camford Books. ISBN: 9781898563860.

[6] Timpe, A. (1912) 'Die Torsion von Umdrehungskörpern', Mathematische Annalen, 71, pp. 480509. doi: 10.1007/BF01456805.

[7] Bramble, J. H. (1959) 'The Thick Elastic Spherical Shell Under Concentrated Torques', Proceedings of the London Mathematical Society, 3(4), pp. 492-502. doi: 10.1112/plms/s39.4.492.

[8] Pöschl, Th. (1922) 'Bisherige Lösungen des Torsionsproblems für Drehkörper', Zeitschrift für Angewandte Mathematik und Mechanik (ZAMM), 2(2), pp. 137-147. doi: 10.1002/zamm.19220020207.

[9] Arutyunyan, N. H., Abramyan, B. L. (1963) 'Torsion of Elastic Bodies', (in Russian) Moscow: Fizmatgiz.

[10] Föppl, A. (1905) 'Die Torsion von runden Stäben mit veranderlichem Durchmesser', Sitzungsb. Bayer. Akad. wiss. München, 35, pp. 249-305.

[11] Ecsedi, I. (1985) 'A special case of the problem of hollow-core solids of revolution', Acta Technica Academiae Scientiarum Hungarica, 98(3-4), pp. 275-293.

[12] Lekhnitskii, S. G. (1971) 'Torsion of Anisotropic and Non-homogeneous Bars', (in Russian) Moscow: Fizmatgiz.

[13] Kamke, E. (1951) 'Differentialgleichungen. Lösungsmethoden und Lösungen I-II.' Leipzig: Akademische Verlagsgesellschaft Gest and Portig K.-G., ISBN: 978-3663120582.

[14] Forsyth, A. R. (1929) 'A Treatise on Differential Equations.' London: 6th Ed., MacMillan. 